\title{
A SIMPLIFICATION OF D'ALARCAO'S IDEMPOTENT SEPARATING EXTENSIONS OF INVERSE SEMIGROUPS
}

\author{
CONSTANCE C. EDWARDS \\ Department of Mathematical Sciences \\ Indiana University-Purdue University at Fort Wayne \\ Fort Wayne, Indiana 46805 U.S.A. \\ (Received August 29, 1977)
}

ABSTRACT, In [2] D'Alarcao states necessary and sufficient conditions for the attainment of an idempotent-separating extension of an inverse semigroup. To do this D'Alarcao needed essentially three mappings satisfying thirteen conditions. In this paper we show that one can achieve the same results with two mappings satisfying eight conditions.

KEY WORDS AND PHRASES. Inverse semigroup and idempotent-separating extension. AMS (MOS) SUBJECT CLASSIFICATION (1970) CODES. 20MIO

\section{THE CONSTRUCTION.}

Unless otherwise mentioned, the terminology and notation of Clifford and Preston [1] shall be assumed. The reader is referred to [2] for all other undefined terms.

LEMMA I. If $A$ and $B$ are inverse semigroups and $f$ is a homomorphism of $A$ onto $E(B)$ which is one-to-one on $E(A)$, then 
(i) af $=\left(a^{-1} a\right) f$ for all a $\varepsilon A$;

(ii) $a^{-1} g a=g a^{-1} a$ for $a \mid l a \in A$ and $g \in E(A)$; and

( $i$ ii) ga $=$ ag for all a $\varepsilon A$ and $g \in E(A)$.

PROOF. (i) Since af $\varepsilon E(B)$, by Lallement's result [3], there exists e $\varepsilon E(A)$ such that af $=$ ef. Consequently, ef $=e^{-1} f=(e f)^{-1}=(a f)^{-1}=a^{-1} f$, and thus af $=$ ef $=\left(a^{-1} f\right)(a f)=\left(a^{-1} a\right) f$.

(ii) By part (i), for each $g \in E(A)$ and a $\varepsilon A$, $\left((g a)^{-1} g a\right) f=(g a) f=g f \cdot a f=g f \cdot\left(a^{-1} a\right) f=\left(g a^{-1} a\right) f$. Since $f$ is one-toone on $E(A)$, we have $a^{-1} g a=(g a)^{-1} g a=g a^{-1} a$.

(iii) By part $(i \mathrm{i})$, ga $=a\left(a^{-1} g a\right)=a\left(g a^{-1} a\right)=a g$. This completes the proof of the lemma.

Let $A$ and $B$ be inverse semigroups and let $f$ be a homomorphism of A onto $E(B)$ which is one-to-one on $E(A)$. (The existence of such an $f$ implies that A is a semilattice of groups; see e.g. [4].) Suppose there is a mapping $w$ of $B \times B$ into $A$ (denoted by $(b, c) w=b^{C}$ ) and that for each $b \in B$ there is a mapping $U_{b}$ of $A$ into $A$ (denoted by $a U_{b}=a^{b}$ ) such that:

(PI) For each $b, c \in B, b^{c} \varepsilon\left(c^{-1} b^{-1} b c\right) f^{-1}$.

(P2) For each $b \in B, U_{b}$ is a homomorphism.

(P3) If $b, c \in B$ and $a \varepsilon\left(b^{-1} b\right) f^{-1}$, then $a^{c} \varepsilon\left(c^{-1} b^{-1} b c\right) f^{-1}$.

(P4) $(b c)^{d}\left(b^{c}\right)^{d}=b^{c d} c^{d}$ for $a \mid l b, c, d \varepsilon B$.

(P5) If $b \in E(B)$ and $a \in A$, then $a^{b}=$ ea where $\{e\}=E(A) \cap b f^{-1}$.

(P6) For each $b \in B,\left(b b^{-1}\right)^{b}=e$ where $\{e\}=E(A) \cap\left(b^{-1} b\right) f^{-1}$.

(P7) $b^{c}\left(a^{b}\right)^{c}=a^{b c} b^{c}$ for $a \mid l a \varepsilon A$ and $b, c \varepsilon B$.

(P8) For each $b, c \in E(B), b^{c}=$ eg where $\{e\}=E(A) \cap b f^{-1}$ and

$$
\{g\}=E(A) \cap c f^{-1} \text {. }
$$

Let $S^{*}=\left\{(b, a): b \in B\right.$ and $\left.a \in\left(b^{-1} b\right) f\right\}$. Let equality in $S^{*}$ be defined in the usual manner and let multiplication be defined by $(b, a)(c, p)=\left(b c, b^{c} a^{c} p\right)$. 
Moreover, let $\gamma: S^{*} \rightarrow B$ be defined by $(b, a) \gamma=b$.

THEOREM 1. Under the above conditions, $\left(S^{*}, \gamma\right)$ is an idempotent-separating extension of $A$ by B. Moreover, $\left.r\right|_{A}=f$.

PROOF. Note that since fof ${ }^{-1}$ is idempotent separating, fof ${ }^{-1} \subseteq \mathbb{H}$. Consequently, if $e \varepsilon E(A)$ and $e$, $a \varepsilon\left(b^{-1} b\right) f^{-1}$, then ea $=a=a e$.

As is shown in [2], closure in $S^{*}$ follows from $(P I)$ and $(p 3)$ and, associativity follows from (P2), (P4) and (P7).

Our proof that $S^{*}$ is regular is the same as that found in [2] but is more informative. Let $(b, a) \in S^{*}$ and let $x=\left(a^{b^{-1}}\right)^{-1}\left(b^{b^{-1}}\right)^{-1}$. By $(P \mid)$ and $(P 3),\left(\left(a^{b^{-1}}\right)^{-1}\right) f=b b^{-1}=\left(\left(b^{b^{-1}}\right)^{-1}\right) f$. Consequently $\left(b^{-1}, x\right) \in S^{*}$ since $x f=\left(\left(a b^{-1}\right)^{-1}\left(b^{b^{-1}}\right)^{-1}\right) f=b b^{-1}$. Also, by (PI) and

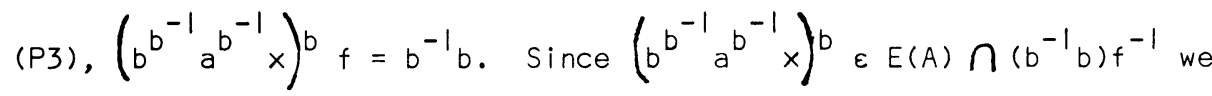
have $\left(b^{b^{-1}} a^{b^{-1}} x\right) b a=a$. It now follows from $(P 6)$ that $(b, a)\left(b^{-1}, x\right)(b, a)=\left(b,\left(b b^{-1}\right),\left(b^{-1} a^{b^{-1}} x\right) b\right)=(b, e a)=(b, a)$ since $\{e\}=E(A) \cap\left(b^{-1} b\right) f^{-1}$. Consequently, $S^{*}$ is regular.

As shown in [2], it follows from $(P 5)$ and $(P 6)$ that if $(g, e) \in E\left(S^{*}\right)$ then $g \varepsilon E(B)$ and $\{e\}=E(A) \cap g f^{-1}$. Consider the subset $A^{*}=\{(b, a): b \varepsilon E(B)\} \subseteq S^{*}$ which contains $E\left(S^{*}\right)$. Let $\delta: A^{*} \rightarrow A$ by $(b, a) \rightarrow a$. To show $\delta$ is a homomorphism, let $(b, a),(c, p) \varepsilon A^{*}$. Then $a=$ ea and $p=g p$ where $\{e\}=E(A) \cap b f^{-1}$ and $\{g\}=E(A) \cap c f^{-1}$. By $(P 8),(P 5)$ and lemma $\mid i i i,(b, a)(c, P)=\left(b c, b^{c} a^{c} p\right)=$ $=(b c$, eg $\cdot g a \cdot p)=(b c$, ea $\cdot g p)=(b c, a p)$. Thus $\delta$ is a homomorphism which obviously has $A$ for its range. It follows from the definitions of $S^{*}$ and $\delta$ that $\delta$ is one-to-one. Consequently the idempotents of $S^{*}$ commute since $A^{*}$ is isomorphic to the inverse semigroup $A$. We now have that $S^{*}$ is an inverse semigroup. 
It is obvious that $\gamma$ is a homomorphism of $S^{*}$ onto $B$ and $\{E(B)\} \gamma^{-1}=A^{*}$. If $(b, a),(c, p) \varepsilon E\left(A^{*}\right)$ such that $(b, a) \gamma=(c, p) \gamma$, then $b=c$ and $a f=b=c=p f$. Since $a, p \in E(A), a=p$. Consequently $\gamma$ is one-to-one on $E\left(A^{*}\right)$. It is obvious that $\left.\gamma\right|_{A^{*}}=f$. Since $A^{*}=A$, this completes the proof of the theorem.

\section{THE STRUCTURE THEOREM.}

Since our two mappings and eight properties are the same as, but fewer than, those used by D'Alarcao, the following theorem has been proven by D'Alarcao ([2], Theorem 2).

THEOREM 2. Let $A$ and $B$ be inverse semigroups and let $(S, f)$ be an idempotent-separating extension of $A$ by $B$. Then for each $b \varepsilon B$ there exists a mapping $U_{b}$ of $A$ into $A$ (denoted by $a U_{b}=a^{b}$ ) and there exists a mapping $w$ of $B \times B$ into $A$ satisfying $(P \mid)$ through (P8). Moreover, $S$ is isomorphic to $S^{*}$.

\section{REFERENCES}

1. Clifford, A. H. and G. B. Preston The Algebraic Theory of Semigroups, Vol. I, Math Surveys of the American Math. Soc. 7 (Providence, RI, 1961).

2. D'Alarcao, H. Idempotent-Separating Extensions of Inverse Semigroups, J. Aust. Math. Soc. 9 (1969) $211-17$.

3. Lallement, G. Congruences et Équivalences de Green sur un Demi-groupe Régulier, C. R. Acad. Sc. Paris Ser A 262 (1966) 6/3-6.

4. Preston, G. B. Inverse Semi-groups, J. of the London Math. Soc. $\underline{29}$ (1954) 396-403. 


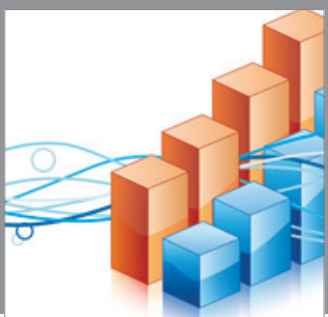

Advances in

Operations Research

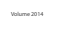

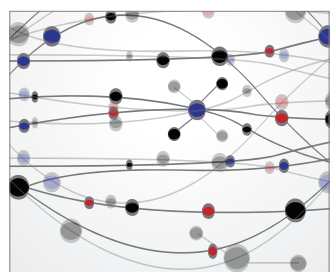

\section{The Scientific} World Journal
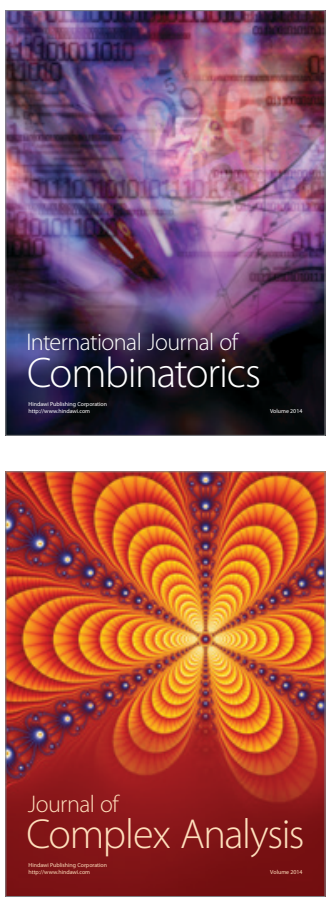

International Journal of

Mathematics and

Mathematical

Sciences
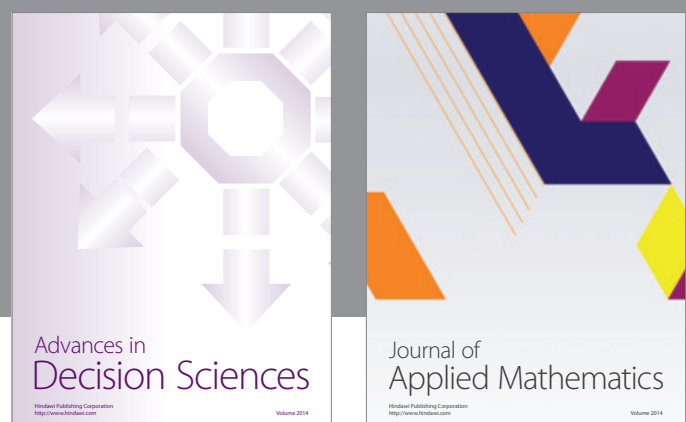

Journal of

Applied Mathematics
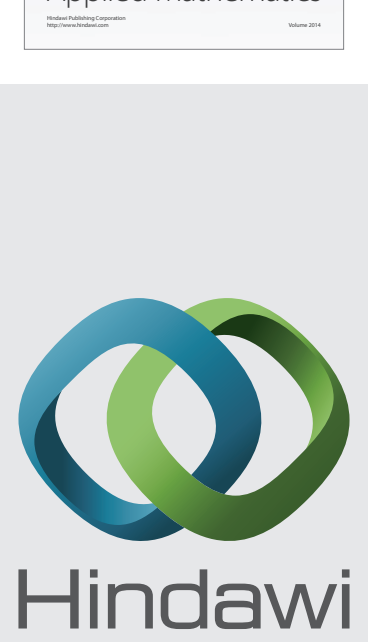

Submit your manuscripts at http://www.hindawi.com
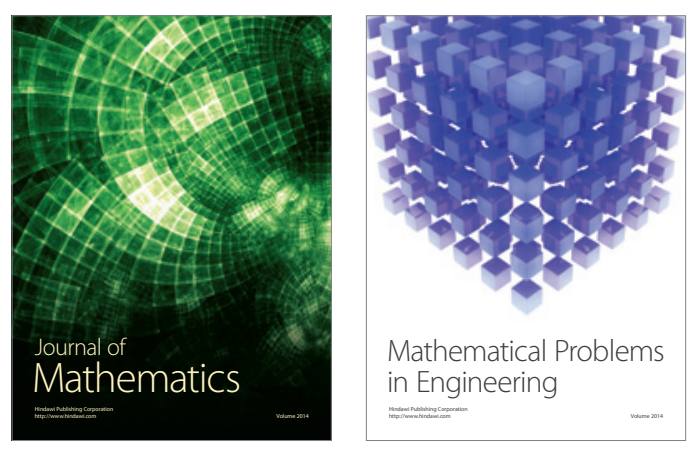

Mathematical Problems in Engineering
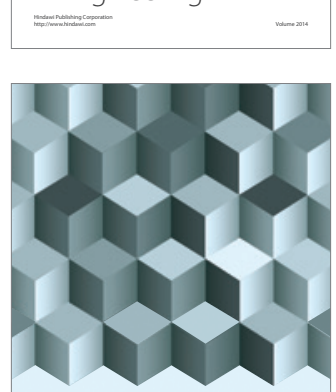

Journal of

Function Spaces
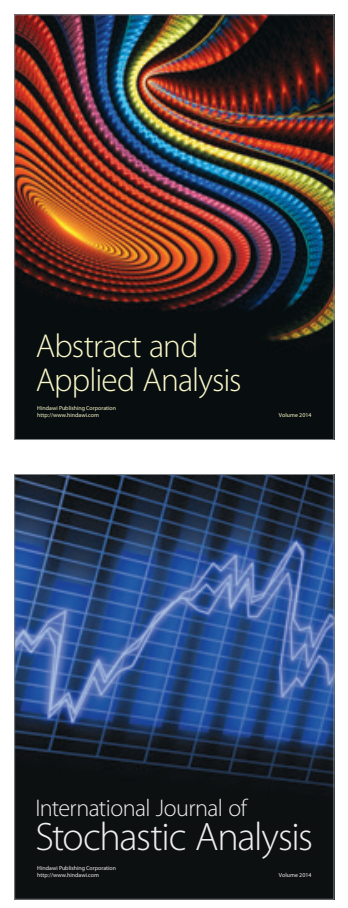

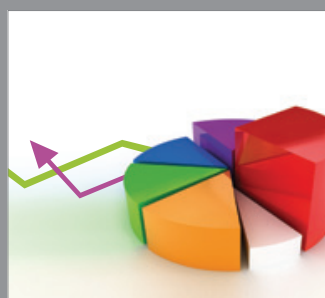

ournal of

Probability and Statistics

Promensencen
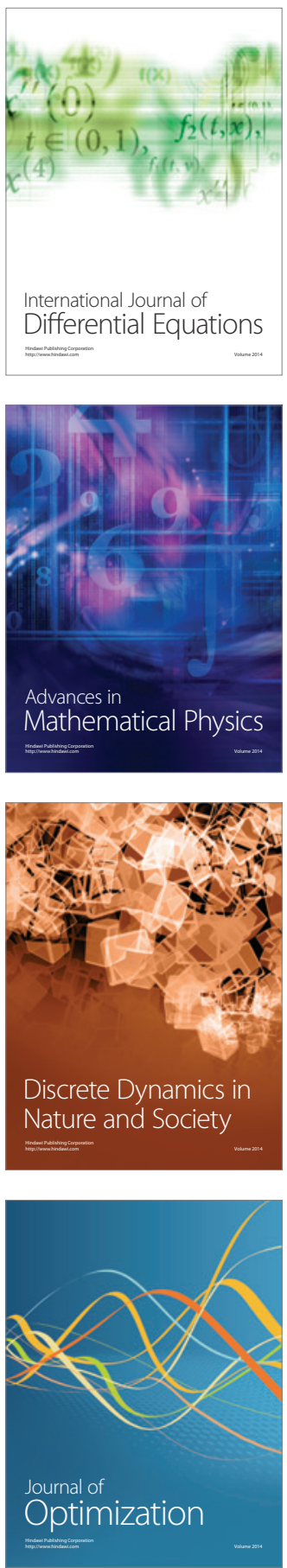